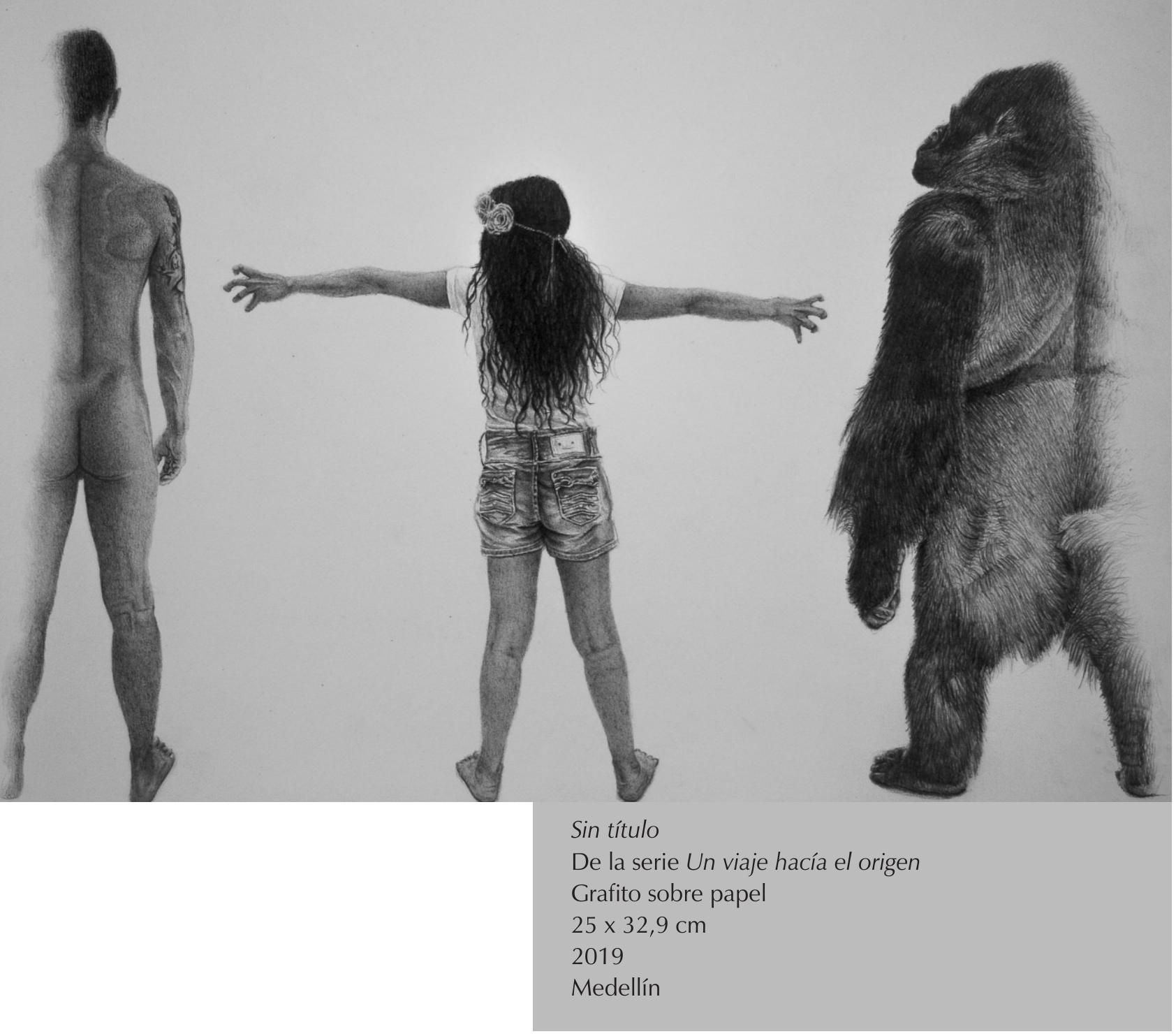




\title{
La movilización internacional de las ideas. Enfoques, conceptos y actores en el estudio de la transferencia y adaptación de políticas públicas*
}

\author{
Carlos Wladimir Gómez Cárdenas (Colombia)**
}

\section{Resumen}

Este artículo desarrolla la discusión de la literatura en torno a los enfoques, conceptos y actores en los procesos de transferencia de políticas públicas, profundizando en el rol que desempeñan actores expertos en la movilización internacional de las ideas, quienes, a pesar de su centralidad en los procesos de transferencia y adaptación de políticas públicas, han sido poco estudiados en nuestro contexto. Dada la relevancia de la temática, el artículo presenta los principales aportes conceptuales y perspectivas de análisis del fenómeno político de la movilización internacional de las ideas, entendido como procesos de transferencia y adaptación de políticas públicas. La revisión de la literatura es pertinente para el desarrollo de nuevas investigaciones en el campo del análisis de las políticas públicas que vinculen como perspectiva teórica la transferencia de políticas públicas.

\section{Palabras clave}

Políticas Públicas; Transferencia; Difusión; Aprendizaje; Movilidad; América Latina.

Fecha de recepción: febrero de 2019 • Fecha de aprobación: julio de 2019

\section{Cómo citar este artículo}

Gómez Cárdenas, Carlos Wladimir. (2019). La movilización internacional de las ideas. Enfoques, conceptos y actores en el estudio de la transferencia y adaptación de políticas públicas. Estudios Políticos (Universidad de Antioquia), 56, pp. 41-65. DOI: 10.17533/udea.espo.n56a03

\footnotetext{
* El presente artículo de revisión se deriva de la tesis doctoral Actores expertos en la transferencia y adaptación de políticas de reforma administrativa en argentina (1991-2008), cuya investigación finalizó en 2016 en la Universidad Nacional de General San Martín, Argentina.

** Politólogo. Especialista en Administración Pública. Magíster en Políticas Públicas. Doctor en Ciencia Política. Profesor asociado y subdirector académico del Instituto de Educación y Pedagogía, Universidad del Valle, Colombia. Correo electrónico: carlos.w.gomez@correounivalle.edu.co - Orcid: 0000-0002-1656-5343 - Google Scholar: https://scholar.google.es/citations?hl=es\&user=oEL2V4kA AAAJ
} 


\title{
The International Mobilization of Ideas. Approaches, Concepts and Actors in the Study of Transfer and Adaptation of Public Policies
}

\begin{abstract}
This review article discusses the approaches, concepts, and actors in public policy transfers. The discussion, however, focuses on the role played by experts in the international mobilization of ideas, who, despite of their central role in transferring policies, have been little studied in our context. Due to the importance of this matter, the article presents the main conceptual contributions and analysis of perspectives about the political phenomenon of international mobilization of ideas understood as processes of public policy transfer. The literature review is relevant for the development of a new field of research on public policy analysis that includes public policy transfers as a theoretical perspective.
\end{abstract}

\section{Keywords}

Public Policies; Policy Transfer; Diffusion; Learning; Mobility; Latin America. 


\section{Introducción}

La movilización internacional de las ideas es un fenómeno político que viene ganando relevancia dentro de las ciencias sociales. A partir de la década de 1990, investigadores en ciencia política, administración pública y políticas públicas han prestado mayor atención al papel de las ideas y de los actores participes en los procesos de diseño institucional. Dentro de la literatura de las ciencias sociales, dicho movimiento se asemeja a los procesos de transferencia y adaptación de políticas públicas — policy transfer-. Para la comprensión de este fenómeno se han aportado una gran pluralidad de conceptos y enfoques dentro de una discusión relacionada con el rol central que desempeñan las ideas en los diseños institucionales. La literatura sobre la temática es cada vez más robusta en cuanto al papel de las ideas. En la distinción conceptual se destaca la diferenciación aportada por diversos autores entre los términos transferencia, difusión, aprendizaje y movilidad de ideas o políticas. Los enfoques o perspectivas para comprender dicho fenómeno internacional de movilización de las ideas se ubican entre «las comunidades de política», «las comunidades epistémicas» las «redes de transferencia» y la «transferencia acotada».

Los diversos avances sobre la comprensión de este fenómeno político de escala global vinculan a los diversos procesos de transferencia y adaptación de ideas o políticas y actores de diversa naturaleza. La literatura destaca a actores de cuya expertise o conocimiento técnico acumulado juega a favor la movilización internacional de las ideas.

Dada la importancia de este fenómeno político y la necesidad de su comprensión en nuestro contexto latinoamericano, este artículo revisa los principales aportes teóricos y conceptuales sobre la materia. La distinción conceptual que se propone y la presentación de los enfoques de análisis, con especial atención al rol de los actores expertos, bien podrían inspirar nuevas investigaciones sobre la materia, hoy escasas, en nuestro ámbito académico. La revisión cobra importancia toda vez que esta serie de conceptos y enfoques ayudan a describir, interpretar y explicar de mejor manera los cambios político-administrativos o institucionales que acontecen en el contexto latinoamericano. 
Particularmente, en dicho contexto latinoamericano y colombiano, los conceptos y enfoques vinculados a la transferencia de políticas públicas que se presentan en este artículo nutren las perspectivas teóricas de análisis para la formulación de las políticas públicas. Esta particular área de desarrollo académico viene ganando terreno en la comprensión de la idea del «Estado en acción». Los analistas de políticas públicas de la región, especialmente en Colombia, pueden complementar lecturas amparadas en modelos de toma de decisiones racionalistas o institucionalistas de los distintos procesos de formulación de políticas públicas, incorporando la perspectiva teórica de la transferencia de políticas públicas, máxime en un contexto donde buena parte de las decisiones de política son transferidas y adaptadas de contextos foráneos.

\section{1. ¿Qué es la trasferencia y la adaptación de políticas públicas?}

La transferencia de políticas - policy transfer- es el proceso por el cual los conocimientos construidos -ideas- de políticas, diseños administrativos e instituciones de una comunidad política son utilizados en la adopción y en el desarrollo de nuevas políticas en otra comunidad.

[ 44 ] Estudiar la transferencia de políticas implica analizar la interdependencia entre procesos y comunidades políticas (Dolowitz, Hulme, Nellis y $\mathrm{O}^{\prime} \mathrm{Neal}$, 2000; Dolowitz y Marsh, 1996; 2002). El término transferencia de políticas fue tempranamente utilizado en campos diferentes a la ciencia política y las políticas públicas. El derecho comparado fue la disciplina que prístinamente empleó tal concepto para dar cuenta del trasplante de normas de tipo legal de un contexto a otro. Alan Watson (1974) fue el pionero en esta materia (De Jong, Lalenis, y Mamadouh, 2002).

En el campo de la ciencia política y específicamente en el terreno del policy analisys, Richard Rose (1991; 1993), Colin Bennett y Michael Howlett (1992), David Dolowitz y David Marsh (1996), Martin de Jong (1999) y Mark Evans y Jonathan Davies (1999) fueron los iniciadores de toda una empresa investigativa que consideraba la posibilidad de transferir otro tipo de instituciones más allá de leyes o normas de tipo legal (véase cuadro 1). A partir de la década de 1990 se comenzaron a considerar nuevas instituciones susceptibles de ser transferidas de un contexto político a otro. 
La movilización internacional de las ideas. Enfoques, conceptos...

Cuadro 1. Preguntas centrales en los estudios de transferencia de políticas públicas.

\begin{tabular}{|l|l|}
\hline \multicolumn{1}{|c|}{$\begin{array}{c}\text { Interrogantes en los procesos } \\
\text { de transferencia de políticas }\end{array}$} & Nivel y aspectos relevantes en la indagación \\
\hline $\begin{array}{l}\text { ¿Por qué los actores participan en la } \\
\text { transferencia de políticas? }\end{array}$ & Intereses y motivaciones. \\
\hline $\begin{array}{l}\text { ¿Quiénes son los actores clave } \\
\text { involucrados en el proceso de la } \\
\text { transferencia de políticas? }\end{array}$ & $\begin{array}{l}\text { Políticos, burócratas, legislaturas, agencias } \\
\text { supranacionales, gobiernos nacionales y } \\
\text { subnacionales, cuerpos administrativos, } \\
\text { empresas privadas, partidos políticos, medios de } \\
\text { comunicación, redes de expertos. }\end{array}$ \\
\hline ¿Qué se transfiere? & $\begin{array}{l}\text { Instituciones, actitudes, ideas, políticas, } \\
\text { programas, proyectos, instrumentos. }\end{array}$ \\
\hline ¿De dónde se extraen las lecciones? & Contextos internacionales, nacionales y locales. \\
\hline $\begin{array}{l}\text { ¿Cuáles son los diferentes grados de } \\
\text { transferencia? }\end{array}$ & $\begin{array}{l}\text { Relación entre el proceso de transferencia de las } \\
\text { políticas con los éxitos o fracasos de la política. }\end{array}$ \\
\hline $\begin{array}{l}\text { ¿Qué limita o facilita el proceso de la } \\
\text { transferencia de políticas? }\end{array}$ & $\begin{array}{l}\text { Recursos y apoyos políticos, económicos, } \\
\text { sociales y culturales. }\end{array}$ \\
\hline
\end{tabular}

Fuente: elaboración propia a partir de Dolowitz y Marsh (2000).

Adicionalmente, se consideró explicar el papel desempeñado por actores más allá de las tradicionales legislaturas, políticos profesionales y burócratas. Agencias supranacionales, gobiernos nacionales y subnacionales, cuerpos administrativos, empresas privadas, partidos políticos, medios de comunicación, redes de expertos, entre otros, llamaron la atención dentro del proceso (Bozeman, 2000; Stone, 2000; 2001; 2004; Dolowitz y Marsh, 2002; Nickson, 2002; Rubio, 2002; Pollitt, 2003; Evans, 2004; Pal, 2006; Reinholde, 2006; Levi-Faur y Vigoda-Gadot, 2006; Culebro, 2008; Dussauge, 2009; 2010).

A pesar de las potencialidades de los procesos de transferencia de políticas públicas, cuando Ilegan a su etapa de adaptación en la sociedad de destino son frecuentemente resistidos o rechazados. Esta situación sugiere ver este tipo particular de procesos como algo complejo, en lugar de una simple imitación u homologación de instituciones, ideas, políticas, programas o instrumentos (véase cuadro 2). Como en un trasplante quirúrgico, el nuevo órgano trasplantado es rechazado por el nuevo cuerpo, las instituciones pueden sufrir el mismo destino si no se atienden los objetivos del proceso y las condiciones del nuevo contexto (De Jong et al., 2002). 
Cuadro 2. Factores intervinientes en los procesos de transferencia de políticas.

\begin{tabular}{|l|l|}
\hline \multicolumn{1}{|c|}{ Factor } & \multicolumn{1}{c|}{ Escenarios y trampas potenciales } \\
\hline Sistema Político & $\begin{array}{l}\text { Autonomía de los importadores, complejo conjunto de } \\
\text { relaciones. }\end{array}$ \\
\hline Cultura & $\begin{array}{l}\text { Idiomas involucrados - sentidos y significados-, } \\
\text { principios y valores -origen y destino-, desacople } \\
\text { cultural. }\end{array}$ \\
\hline Estrategia de gestión & Complejidad de la técnica o del enfoque. \\
\hline Naturaleza de las tareas primarias & Calidad del análisis del (los) problema(s) a enfrentar. \\
\hline Patrones institucionales & Adaptación y ajuste institucional. \\
\hline
\end{tabular}

Fuente: elaboración propia a partir de De Jone et al. (2002) y Pollitt (2005).

Los procesos de transferencia de políticas están lejos de ser interpretados como algo simple, no se trata de la sencilla compra de un producto estándar, generalmente implican una significativa adaptación, en tanto transformación de la «cuestión» transferida; además, traen consigo algo más que solo el instrumento o herramienta que se importa. Supuestos, normas, prerrequisitos y, por supuesto, consecuencias inesperadas están en juego dentro de la transferencia de políticas (Pollitt, 2005).

De esta manera, se hace necesario vincular todo proceso de transferencia a un proceso de adaptación. No es posible entender por separado estas dos complejas instancias, ni mucho menos dejar de reconocer su naturaleza multiactoral, multicultural y multirelacional, que por supuesto involucra actores que tienen distinta autoridad, poder, experiencia y expertise. Transferencia y adaptación de políticas resultan siendo dos caras de una misma moneda.

\section{El estudio de los procesos de transferencia y adaptación de políticas públicas}

El estudio de los procesos de transferencia y adaptación de políticas es un campo de investigación relativamente reciente que ha experimentado un auge significativo en los últimos años. En la última década ha habido un creciente aumento de la literatura en ciencias sociales que directa o indirectamente viene discutiendo y analizando los procesos de convergencia, difusión, transferencia y adaptación de políticas. 
La manera en la cual las ideas, normas e instituciones internacionales que se desarrollan en un país o conjunto de países viajan a otros contextos y sistemas políticos ha atraído la atención de diversos sectores, especialmente de aquellos relacionados con la implementación de nuevas políticas públicas. Este especial interés ha sido reforzado por un conjunto de presiones de orden técnico que influyen cada vez más en las decisiones de los Estados, así como por el ya mencionado avance de las nuevas tecnologías de información que incrementan losflujosdecomunicaciónentrepersonasypaíses(Culebro,2008).

Interrogantes como: ¿por qué y cuándo los actores participan en la transferencia de políticas?, ¿quién transfiere las políticas?, ¿qué se transfiere?, ¿de dónde son las lecciones aprendidas?, ¿existen diferentes grados de transferencia?, ¿qué actores participan en la transferencia de políticas y cómo afecta esto las decisiones y los procesos de transferencia de políticas?, ¿qué limita la transferencia de políticas?, ¿cómo pueden los investigadores empezar a demostrar la ocurrencia de la transferencia de políticas? y ¿cómo puede la transferencia de políticas ayudarnos a comprender los fracasos de la política?, han proporcionado un verdadero marco para la investigación del movimiento de políticas en la comunidad internacional (Dolowitz et al., 2000). En estos términos, la transferencia de políticas públicas es un fenómeno central en el campo de la ciencia política y en particular en la administración pública, donde existen muchos ejemplos de reformas e innovaciones de políticas que han sido transferidos de un contexto internacional, regional o nacional a otro (Conde, 2005).

Los estudios sobre transferencia de políticas públicas han enfatizado en el elemento intencional como aspecto definitorio del proceso, sustentándose en la presunción implícita de que la transferencia es el resultado de un proceso de aprendizaje político y que la élite política del país receptor es el actor principal en el proceso de adaptación endógena de las políticas. Aparece, en este sentido, un sesgo que ha enfatizado en el estudio de los actores tradicionales u oficiales de la transferencia -burócratas y políticos profesionales-, desatendiéndose otros posibles actores no oficiales igualmente claves dentro del proceso -académicos, expertos y organizaciones no oficiales- (Stone, 2004). A su vez, la mayoría de estudios adoptan un enfoque clásico para el análisis del proceso decisional basado en el modelo secuencial o por etapas del proceso político, y explican la decisión de importar a partir de modelos del cambio en la agenda política interna (Rubio, 2002). 
A pesar del desarrollo significativo que ha experimentado la literatura sobre transferencia de políticas públicas, esta sigue adoleciendo de ciertas limitaciones teóricas para el análisis y la explicación de los fenómenos de interdependencia política. El principal problema que presenta es su desconexión con los grandes debates o cuestiones que orientan la investigación en ciencia política. La mayoría de estudios sobre la transferencia son estudios empírico-descriptivos, en la medida en que se orientan principalmente a demostrar si se ha producido o no un proceso de transferencia sin que el proceso de búsqueda y análisis de información esté guiado por un marco conceptual definido, con hipótesis de investigación específicas sobre qué factores han motivado los procesos de transferencia (Rubio, 2002).

En consecuencia, la literatura podría beneficiarse de investigaciones que, dejando a un lado la premisa de la transferencia, centren su atención en otras preguntas que ofrezcan una imagen más completa de estos procesos. Estudios que intenten determinar no solo el cómo, sino el porqué de los factores que motivaron los diferentes procesos de transferencia y quizás, lo más importante, cómo y por qué influyen los diversos actores — diferentes a los tradicionales burócratas y políticos profesionales- a lo largo del proceso (Dussauge, 2009; 2010).

El conocimiento que tenemos en la materia se nutriría de investigaciones que centren su atención en la etapa del proceso que antecede la transferencia de las políticas y en la etapa posterior, lo que implica la adaptación de la política transferida, determinando el rol específico y las motivaciones de los actores en la movilización internacional de las ideas. Las investigaciones podrían indagar sobre cómo y por qué actores tradicionales —burócratas y políticos profesionales- y no tradicionales -expertos, académicos, mediadores sociales- influyen en la transferencia y adaptación de las políticas públicas. Indagar sobre el proceso, más allá del momento de la transferencia de políticas, avanzando no solo en mostrar su intencionalidad, implica obtener una imagen más completa de la transferencia, toda vez que reconoce un antes, un durante y un después del proceso, si se considera la idea de que toda transferencia implica adaptación.

En el contexto latinoamericano, el enfoque de transferencia de políticas públicas ha ayudado a comprender mejor los cambios políticoadministrativos o institucionales. Una de las temáticas más abordada en 
la región bajo el enfoque de la transferencia de políticas públicas son los programas de transferencia monetaria condicionada (Valencia, 2008; Fiszbein y Schady, 2009; Puello-Socarrás y Gunturiz, 2013; Osorio, 2015; Villatoro, 2005; Corbella y Calle, 2017).

Los trabajos de José Francisco Puello-Socarrás y María Angélica Gunturiz (2013) y Cecilia Osorio (2015), destacan el rol de los organismos internacionales en la transferencia de políticas públicas bajo los programas de transferencia monetaria condicionada en América Latina. Para PuelloSocarrás y Gunturiz (2013), organismos internacionales como el Banco Mundial y el Banco Interamericano de Desarrollo han sido los actores más influyentes en el diseño, implementación, evaluación e institucionalización de los programas de transferencia monetaria condicionada en la región. Por su parte, Osorio (2015) muestra cómo se difundieron estos programas a escala planetaria muy rápidamente en la primera década del siglo XxI gracias al rol de los organismos internacionales. Para 2010, 29 países -17 Latinoamericanos- habían implementado el programa de transferencia monetaria condicionada como su principal estrategia para mitigar la pobreza. En cuanto a los actores internacionales, es preciso identificar, más allá del Banco Mundial y el Banco Interamericano de Desarrollo, organismos internacionales dependientes de la Organización de Naciones Unidas Comisión Económica para América Latina y el Caribe- y regionales, como la Organización de Estados Americanos.

Estos aportes dejan ver los avances en la materia en América Latina bajo el enfoque de transferencia de políticas públicas, con especial atención al rol que han desempeñado en estos procesos los diferentes organismos internacionales. La comprensión del movimiento internacional de las ideas en la región ha sido facilitada por el policy transfer.

\section{Conceptos y enfoques alrededor de la transferencia de políticas públicas}

\subsection{Movilización internacional de las ideas: entre la transferencia, la difusión, el aprendizaje y la movilidad}

Los conceptos transferencia, difusión, aprendizaje y movilidad son utilizados muchas veces de manera indistinta como sinónimos cuando nos referimos al movimiento internacional de las ideas. Sin embargo, la mayor 
parte de la literatura distingue el uso de los términos transferencia — policy transfer-, difusión — policy diffusion-, aprendizaje — policy learning- y movilidad - policy mobilities-.

En cuanto a la transferencia - policy transfer-, la referencia más utilizada en la literatura es la de Dolowitz y Marsh (2000), que definen la transferencia como «el proceso por el cual el conocimiento acerca de las políticas, arreglos administrativos, instituciones e ideas en un sistema político (pasado o presente) es usado en el desarrollo de políticas, arreglos administrativos, instituciones e ideas en otro sistema político» (Dolowitz y Marsh, 2000, p. 5).

El término de difusión — policy diffusion — tiene similar importancia en la literatura que el de - policy transfer-, incluso algunos investigadores los han catalogado como conceptos complementarios (Marsh y Sharman, 2009). Esta acepción suele utilizarse para definir un fenómeno más de orden general y no de rango medio, tal como apela la transferencia - proceso de decisión política que conlleva la exportación de una política, programa o enfoque de un sistema político a otro-, e implica la dispersión, la diseminación, la propagación de una innovación política, como, por ejemplo, un nuevo

[ 50 ] programa o una reforma institucional en varias jurisdicciones que forman parte de un mismo contexto nacional o internacional (Rubio, 2002). La difusión puede ser definida como «el proceso por el cual una innovación es comunicada a través de ciertos canales a través del tiempo entre los miembros del sistema social. Es un tipo especial de comunicación en que los mensajes se refieren a nuevas ideas» (Rogers, 1995, p. 5).

Los estudios de difusión de las políticas tienden a resolver interrogantes vinculados a los mecanismos y patrones de la difusión. ¿Por qué algunas medidas se difunden más rápido que otras?, ¿por qué varían tanto los patrones regionales de difusión de políticas?, ¿qué mecanismos de difusión tienen la probabilidad de resultar influyentes en las fases de difusión de políticas? y ¿cómo son afectados los patrones de difusión por variables políticas?, son algunos interrogantes centrales dentro de la literatura de la policy diffusion (Meseguer y Gilardi, 2008).

Los conceptos de transferencia - policy transfer- y difusión policy diffusion-, al margen de su posible diferenciación, también pueden entenderse como complementarios. La comprensión de cada sector o área 
de política pública puede aumentar en la medida en que se combinen los diversos mecanismos imbricados en la transferencia o en la difusión. Combinar los niveles de análisis centrado en la estructura - privilegiado por los estudios de difusión- y centrado en la agencia — privilegiado por los estudios de transferencia- podría aportar igualmente mayores elementos para la comprensión de estos fenómenos; a su vez, armonizar los hallazgos de patrones propios en la difusión con los rastreos de procesos propios de los estudios de transferencia (Marsh y Sharman, 2009).

En tercer orden, el término aprendizaje - policy learning_, a diferencia de la transferencia y la difusión, opera más en el terreno de lo particular y se refiere al proceso de cognición y de redefinición de intereses por parte de actores locales, con base en el nuevo conocimiento adquirido que afecta las creencias - principios y valores-, así como en las ideas que fundamentan una determinada política (Hall, 1993).

Más allá de esta diferenciación, existe una tendencia en la literatura que vincula el aprendizaje con la transferencia, entendiendo esta última como un proceso de aprendizaje político en sí mismo. Rose (1991) define la transferencia como un proceso de lesson-drawing, en tanto proceso de recogida de información, evaluación y adaptación de las experiencias desarrolladas en otros países, con el objetivo de mejorar la eficacia de las propias políticas. El aprendizaje político se extrae del análisis de las acciones de otros gobiernos que puedan replicarse en contextos propios. Un punto en común en la literatura sobre el aprendizaje político es que hace hincapié en la cognición y la redefinición de los intereses de los actores sobre la base de nuevos conocimientos, el cual afecta las creencias fundamentales y las ideas detrás de la política (Hall, 1993; Levi-Faur y Vigoda-Gadot, 2006).

Por su parte, el concepto de movilidad — policy mobilitieses desarrollado por autores como Magdaléna Hadjiisky, Leslie Pal y Christopher Walker (2017), Jamie Peck y Nik Theodore (2015), quienes resaltan su novedad. A pesar de lo incipiente de los hallazgos bajo esta reciente arista del fenómeno, es una de las más crecientes contribuciones para el análisis de la transferencia de políticas públicas. El término policy mobilities, contextualizado en la actual crisis del neoliberalismo, intenta capturar analíticamente no solo el incremento, sino también la velocidad de la transferencia de políticas públicas, en una era de cada vez mayor interconectividad global. 
Sus desarrollos aportan al entendimiento del fenómeno internacional de movilidad de las ideas y está soportados en las contribuciones de los estudios urbanos y la geografía urbana (McCann, 2017; McCann y Ward 2015; Crivello, 2015; Temenos y Baker, 2015). Elementos vinculados a la producción social de los territorios y las ciudades son discutidos por esta línea de trabajo, con la bondad de introducir en los análisis aspectos del contexto, imbricados con una crisis del neoliberalismo, en la cual los lugares de hechura de las políticas públicas y los propios hacedores, están marcados por fenómenos de interpenetración relacional y mutua interdependencia (Hadjiisky, Pal y Walker, 2017).

Estos cuatro fenómenos hacen parte de un proceso político más complejo de movilización internacional de las ideas, donde transferencia, difusión, aprendizaje y movilidad son vistos como sucesos colectivos fundados en el intercambio de diversos grupos y actores. Para complementar esta conceptualización se revisarán los enfoques desde los cuales se ha explicado dicho movimiento internacional de las ideas.

\subsection{Enfoques en la transferencia de políticas: comunidades políticas, comunidades epistémicas, redes de transferencia y transferencia acotada}

Las perspectivas desde las cuales se ha estudiado el cada vez más creciente movimiento internacional de las ideas varían considerablemente dentro de la literatura. Aparecen vinculados los enfoques de comunidades políticas, comunidades epistémicas, redes de transferencia y el enfoque de transferencia de políticas acotado - bounded policy transfer-.

El primero de los enfoques fue aportado por David Marsh y R. A. W Rhodes (1992a; 1992b). Para estos autores el proceso de transferencia responde a la idea de "comunidad de políticas». Una comunidad se constituye por un número muy limitado de participantes donde inclusive algunos grupos pueden ser excluidos de manera consciente. En dicha comunidad predominan los intereses económicos o profesionales. La interacción es frecuente entre los grupos y se genera con relación a todos los asuntos vinculados con la política pública. Todos los miembros comparten un conjunto de principios y valores básicos y aceptan la legitimidad del resultado, derivados de la concreción de dichos principios y valores. Todos poseen recursos -económicos, políticos, culturales, sociales e 
intelectuales- y la relación básica es de intercambio. A pesar de que un grupo puede dominar a otro, la relación siempre es de suma positiva para que persista la comunidad (Evans y Davies, 1999).

Cuadro 3. Enfoques en la transferencia de políticas.

\begin{tabular}{|l|l|l|l|}
\hline Dimensión & Comunidades políticas & \multicolumn{1}{|c|}{$\begin{array}{c}\text { Comunidades } \\
\text { epistémicas }\end{array}$} & Redes de transferencia \\
\hline Membresía & $\begin{array}{l}\text { Muy limitada. Intereses } \\
\text { económicos y } \\
\text { profesionales }\end{array}$ & $\begin{array}{l}\text { Limitada. Intereses } \\
\text { disciplinares }\end{array}$ & $\begin{array}{l}\text { Muy limitada. Intereses } \\
\text { burocráticos y } \\
\text { tecnocráticos }\end{array}$ \\
\hline Interacción & $\begin{array}{l}\text { Frecuente y de alta } \\
\text { calidad de todos los } \\
\text { grupos en relación a la } \\
\text { política }\end{array}$ & $\begin{array}{l}\text { Poco frecuente. } \\
\text { Negociación y } \\
\text { regateo dentro y entre } \\
\text { comunidades }\end{array}$ & $\begin{array}{l}\text { Frecuente y de alta } \\
\text { calidad de todos los } \\
\text { grupos en relación con la } \\
\text { transferencia de políticas }\end{array}$ \\
\hline Continuidad & $\begin{array}{l}\text { Permanente y } \\
\text { persistente en el tiempo }\end{array}$ & $\begin{array}{l}\text { Acotada. Siempre y } \\
\text { cuando sobreviva la } \\
\text { reputación }\end{array}$ & $\begin{array}{l}\text { Ad hoc. Permanencia } \\
\text { establecida con intención } \\
\text { específica }\end{array}$ \\
\hline Poder & $\begin{array}{l}\text { Equilibrio de poder } \\
\text { entre los miembros. } \\
\text { Influencia se da por } \\
\text { la persistencia y } \\
\text { permanencia de la } \\
\text { comunidad }\end{array}$ & $\begin{array}{l}\text { Elaboración de } \\
\text { políticas determina } \\
\text { la influencia de la } \\
\text { comunidad y su estado } \\
\text { de aceptación }\end{array}$ & $\begin{array}{l}\text { Capacidad de los agentes } \\
\text { de satisfacer objetivos de } \\
\text { políticas de sus clientes }\end{array}$ \\
\hline
\end{tabular}

Fuente: elaboración propia a partir de Evans y Davies (1999).

El segundo de los enfoques fue elaborado por Emanuel Alder y Peter Haas (1992). Este par de autores interpretan la transferencia de políticas como un proceso impulsado por la fuerza en la creencia de principios y causalidades - analíticas y normativas - que actúan como mecanismos de filtro que excluye ciertos aportes. De esta manera, la comunidad epistémica se constituye con un número limitado de participantes que creen en tales principios y causalidades. La membresía en la comunidad aparece vinculada a las disciplinas o profesiones cuyos conocimientos reconocidos sobre políticas les otorga su legitimidad y reputación. Todos sus miembros poseen recursos de conocimiento basados en una relación de intercambio y su participación en la elaboración de políticas determina su influencia y su estado de aceptación (Evans y Davies, 1999). El control sobre la producción de conocimiento e información permite a las comunidades epistémicas exportar sus proyectos de políticas en el ámbito global (Dunlop, 2009). 
En el tercero de los enfoques aportado por Evans y Davies (1999) se entiende la transferencia de políticas bajo la lógica de una «red», donde los principales interesados son los agentes de la transferencia, los políticos y burócratas o funcionarios afectados. La red de transferencia de políticas se teje ad hoc y se encuentra orientada a la acción, se establece en este sentido con la intención específica de impulsar el cambio de políticas. Todos los participantes comparten principios básicos, poseen recursos y responden a una razón de intercambio. El éxito de la red se encuentra soportado en la capacidad de sus miembros de satisfacer el objetivo de política de su cliente (Evans y Davies, 1999).

El cuarto de los enfoques para el estudio de la transferencia de políticas públicas, es aportado por Mauricio Dussauge $(2009 ; 2010)$ (véase cuadro 4), para quien los enfoques de comunidad de políticas, comunidades epistémicas y redes de transferencia se relacionan con dos generaciones iniciales o precedentes de estudios sobre transferencia de políticas. Su «acotado» enfoque lo vincula a una tercera generación de estudios que pretende complementar las variadas perspectivas precedentes de análisis del movimiento internacional de las ideas. Este autor abre la posibilidad de entender la transferencia de políticas en atención a su particular contexto y [ 54 ] los límites que acotan el resultado.

Bajo esta nueva perspectiva, la transferencia de políticas, en lugar de ser concebida como un proceso amplio, abierto y libre en tanto intencional, es visto como un proceso a través del cual se utiliza el conocimiento acerca de las políticas o programas existentes en una jurisdicción a incorporar o desarrollar políticas o programas similares en otra jurisdicción, claramente determinado y limitado por las características y legados políticos, administrativos e institucionales de la jurisdicción importadora (Dussauge, 2009; 2010).

Las variables contextuales y organizacionales -estructuras burocráticas, cultura organizacional, rutinas administrativas, compromisos de política, normas legales- son entendidas aquí como límites que no solo determinan el proceso, sino que ayudan a explicarlo. El conocimiento de las características y valores particulares de una tradición administrativa nacional, la cultura organizacional y los legados políticos, podrían ayudar a comprender, entre otros aspectos, cómo y por qué avanzará el propio proceso de transferencia, o cuáles son los principales desafíos que deberán enfrentar en el futuro próximo los actores centrales de la transferencia. 
Cuadro 4. Enfoque amplio y acotado de transferencia de políticas.

\begin{tabular}{|l|l|l|}
\hline \multicolumn{1}{|c|}{ Factores } & \multicolumn{1}{|c|}{ Policy Transfer } & \multicolumn{1}{c|}{ Bounded Policy Transfer } \\
\hline $\begin{array}{l}\text { Dimensión } \\
\text { temporal }\end{array}$ & $\begin{array}{l}\text { Incierta } \\
\text { Fundamentada en el corto } \\
\text { plazo }\end{array}$ & $\begin{array}{l}\text { Explícita } \\
\text { Basada en una perspectiva de largo plazo } \\
\text { Consideraciones antes y después de la } \\
\text { transferencia }\end{array}$ \\
\hline $\begin{array}{l}\text { Límites de la } \\
\text { transferencia }\end{array}$ & $\begin{array}{l}\text { Técnicos, legales, } \\
\text { administrativos } \\
\text { Preocupaciones por la } \\
\text { compatibilidad }\end{array}$ & $\begin{array}{l}\text { Tradición administrativa: valores y } \\
\text { comportamientos } \\
\text { Estructuras burocráticas } \\
\text { Instituciones políticas } \\
\text { Compromisos políticos } \\
\text { Oportunidades políticas }\end{array}$ \\
\hline $\begin{array}{l}\text { Despliegue de la } \\
\text { transferencia }\end{array}$ & $\begin{array}{l}\text { Incierto } \\
\text { Adaptación de la innovación } \\
\text { Política transferida como } \\
\text { variable independiente }\end{array}$ & $\begin{array}{l}\text { Niveles institucionales } \\
\text { Cambio institucional } \\
\text { Política transferida como variable } \\
\text { independiente o dependiente }\end{array}$ \\
\hline Aprendizaje & Evaluación prospectiva & $\begin{array}{l}\text { Evaluación prospectiva y aprendizaje } \\
\text { adaptativo }\end{array}$ \\
\hline $\begin{array}{l}\text { Origen de las } \\
\text { ideas }\end{array}$ & $\begin{array}{l}\text { Casos relevantes o exitosos } \\
\text { Casos y líderes de referencia }\end{array}$ & $\begin{array}{l}\text { Precedentes poderes coloniales } \\
\text { Países con administración, tradiciones y } \\
\text { vínculos culturales compartidos } \\
\text { Redes de colegaje }\end{array}$ \\
\hline
\end{tabular}

Fuente: elaborado a partir de Dussauge (2010).

La estructura organizativa y los procedimientos del sistema de mérito podrán, de igual forma, ayudar a explicar por qué determinados grupos políticos o actores participan en el proceso de transferencia, con qué grados de influencia y a través de qué canales. Dentro de este enfoque, el marco institucional, así como los patrones históricos de funcionamiento del gobierno, son útiles también para explicar los resultados generales del proceso de transferencia de políticas públicas (Dussauge, 2009; 2010).

Otras de las principales características de este enfoque es la manera como concibe la temporalidad del proceso de transferencia. Explícitamente se concibe como un proceso situado en una línea de tiempo que vincula herencias del pasado, aspectos del presente y situaciones a futuro. De esta forma, los procesos de transferencia se llevarán a cabo dentro límites institucionales heredados del pasado, los cuales existían antes de iniciar el proceso de transferencia. El proceso de transferencia no concluirá inmediatamente después de la transferencia de la política, sino que 
continuará desarrollándose en el futuro, también dentro de ciertos límites (Dussauge, 2009; 2010).

El enfoque acotado de transferencia, en lugar de hacer énfasis exclusivo en una evaluación de la política de tipo retrospectivo para recopilar información relevante y aprender lecciones sobre las experiencias transferidas, añade la posibilidad de realizar un aprendizaje de tipo adaptativo. La idea de aprendizaje adaptativo significa que los actores también tendrán que aprender para ajustar la nueva política a su nuevo entorno institucional (Dussauge, 2009; 2010).

Frente a los orígenes de las ideas o políticas a transferir y diferenciarse de las generaciones precedentes de los estudios de la movilización internacional de las ideas, el enfoque acotado considera que los diferentes agentes de la transferencia, en lugar de revisar buenas prácticas indistintamente en varios contextos internacionales, buscan similitudes con los contextos de destino a fin de captar las ideas que mejor se adapten a sus necesidades. Esto sugiere que la mayoría de las veces los agentes analizarán áreas que han sido tradicionalmente percibidos como similares y no experiencias exitosas per se (Dussauge, 2009; 2010).

La importancia del enfoque propuesto por Dussauge es la de vincular, más allá de las tradicionales variables estructurales, variables de orden contextual y organizacional. De allí la reivindicación de este enfoque que pone en juego aspectos políticos, institucionales y, sobre todo, administrativos, para ayudar a comprender de una mejor forma los procesos de transferencia y adaptación de políticas públicas.

\section{La política de los expertos, rol e influencia en las esferas de decisión}

El resultado político del proceso de movilización internacional de las ideas es su transferencia y adaptación a contextos locales. Una idea es una entidad que representa la manera particular como se ve y entiende el mundo. A pesar de sus potencialidades, las ideas, en tanto representaciones de la realidad, no cobran vida si no logran encarnarse o enraizarse en los contextos propios de la acción gubernamental, donde las políticas públicas enmarcan dichas representaciones. Sin embargo, las ideas, en tanto entidades o representaciones de la realidad, no se trasmiten por sí mismas, 
ni tampoco ejercen influencia abstracta en los diferentes debates políticos. A diferencia de ello, las ideas son trasmitidas por actores - expertos- en contextos institucionales específicos y sirven para formular políticas que pueden resultar incomprensibles si no se consideran las creencias e intereses de quienes las formulan (Sikkink, 2009).

Los actores, en tanto transmisores e intérpretes, desempeñan un importante papel de mediación en los procesos de inserción institucional de las ideas, en la medida en que las mismas ideas no cobran similar significado en todos los contextos. Tales significados emergen de un proceso interpretativo en el cual ciertos actores desempeñan un papel más privilegiado que otros (Sikkink, 2009). Es claro que, a pesar de su importante rol, los expertos no los únicos actores encargados de echar a andar las ideas. El resultado de cualquiera actividad política no depende exclusivamente de la voluntad de los expertos, pero sí de la interacción entre estos y otros actores políticos y sociales. De allí la relevancia de estudiar la interacción entre los expertos y otros actores políticos y sociales, tales como los políticos profesionales, los académicos, los mediadores sociales y los organismos internacionales, dentro de un contexto político determinado (Gutiérrez, 2010).

La literatura cada vez más le asigna a los expertos el rol principal en el proceso de transferencia y adaptación de las ideas o políticas (Dussauge, 2009; 2010; Morresi y Vommaro, 2011; Evetts, Mieg y Felt, 2006). Los expertos son aquellas personas que transitan entre las esferas del saber —de donde emergen - y las esferas del poder — hacia donde se desplazan su influencia-.

La expertise, en tanto conocimiento acumulado o capital intelectual, es un fenómeno global y los expertos son actores cosmopolitas. La actividad adelantada por los expertos, en atención a sus recursos de conocimiento, se trata, en la mayoría de los casos, de procesos de importación o exportación de ideas, que no solo alimentan los procesos de toma de decisiones, sino que amalgaman el campo experto (Morresi y Vommaro, 2011).

La expertise, dentro de una relación social, aparece como un recurso diferenciador de los expertos de los no-expertos. En tanto recurso diferenciador, la expertise es contextual, temporal y dinámica: contextual, ya que un experto es tal en relación con un lugar, una cultura, unas circunstancias y un contexto en particular; temporal, dada la situación en que 
un no-experto puede adquirir expertise y utilizarla en cualquier momento presentándose con más experiencia que otro ya experto; dinámica, ya que la posición relativa de un experto cambia continuamente de la mano de los cambios propios de su campo y contexto (Nunn, 2008).

El estudio del campo experto y de los expertos en tanto actores vinculados a los procesos de transferencia y adaptación de ideas o políticas, gana cada vez más terreno en la literatura de las políticas públicas. Dentro de esta literatura, recientemente han emergido tres estrategias particulares de orden metodológico para abordar el estudio de los expertos. En primer lugar, es posible explicar el campo experto a través del análisis de las trayectorias individuales de sus miembros, realizando un trabajo de reconstrucción sociohistórica de las relaciones y alianzas conformadas por los expertos de un campo en particular, así como sus estrategias, recursos, capitales y apoyos movilizados, para el logro o imposición de un cierto modo de intervención técnica sobre el mundo social (Dezalay y Garth, 2005; Gutiérrez, 2010; Morresi y Vommaro, 2011).

Una segunda posibilidad es poner el foco más que en los expertos, en sus dominios o campos de expertise. Bajo esta estrategia se sugiere abordar

[ 58 ] el terreno en particular del campo experto para dar cuenta de las reglas y jerarquías que establece cada tipo de actividad, las formas de deontología y de justificación política, moral y técnica de los instrumentos movilizados, los espacios de socialización de los nuevos participantes y, sobre todo, los requisitos y criterios de ingreso al campo experto, en particular de nuevos miembros novatos, aprendices o profesionales (Hoffman, 1998; Morresi y Vommaro, 2011).

La tercera opción metodológica, es poner atención no en los expertos ni en su campo, sino ver más allá. Esta opción metodológica implica concentrarse en la producción intelectual u outputs derivados del campo y sus partícipes, además de las posibles actividades de relacionamiento con otros espacios o esferas sociales, tales como la generación de redes y alianzas con distintos grupos sociales (Camou, 1997; 2006; Morresi y Vommaro, 2011; Pinilla, 2012; Barbier et al., 2013).

Poner el foco en los outputs implicaría estudiar los principios y valores ético-políticos de los expertos y su relación con formatos de producción tales como discursos, narrativas, pronunciamientos e intervenciones que 
aparecen en oposición a otros modos particulares de ordenamiento del mundo social. Por su parte, focalizarse en las actividades implicaría develar las estrategias de generación de alianzas y apoyos con otros actores sociales fundamentales para incorporar y procesar temas y problemas bajo la lógica de inclusión de aliados y enemigos en el terreno público que viabilicen la movilización internacional de las ideas (Morresi y Vommaro, 2011).

El conocimiento experto se presenta como racional, neutral y objetivo. Estas facultades de orden simbólico logran, casi de manera automática, el acuerdo entre los conocedores e influyen positivamente en los conflictivos procesos de toma de decisiones. Buena parte de los problemas que se someten a juicio de los expertos por su conocimiento o expertise emergen en contextos burocráticos de decisión administrativa (Huber, 1998). Esta situación en particular ubica a los expertos en un lugar privilegiado de incidencia, ya que su conocimiento derivado de información organizativa acumulada por su experiencia en anteriores pero similares contextos organizativos, convierte automáticamente al conocimiento en poder (Sarfatti, 1988).

Las intervenciones de los expertos sobre el mundo social, además de generarse en contextos organizacionales donde la información acumulada es poder, se encuentran apoyadas en una tendencia a reducir las decisiones políticas a decisiones técnicas. Esta situación refuerza la creencia ideológica común de que existen soluciones técnicas para los siempre diversos y profundos problemas sociales (Sarfatti, 1988; Huber, 1998). La relación entre saber técnico y poder político potencializa las posibilidades de influencia de los expertos en los asuntos públicos. El estrecho vínculo entre ciencia y política armoniza premisas de orden normativo con premisas de orden cognitivo. Los recursos que ofrece la ciencia aplicada son susceptibles de ser incorporados en los procesos de transferencia y adaptación de políticas públicas, generándose espacios donde la expertise individual o colectiva toma parte en la deliberación pública (Pinilla, 2012).

Finalmente, en contexto latinoamericano, la relación entre saber y poder se ha manifestado desde la década de 1970 en un crecimiento del número de expertos como de think tanks, centros de investigación, fundaciones privadas y empresas, por lo general ligadas a áreas económicas y financieras tras la expansión del proyecto neoliberal. Comprender dicha relación entre conocimiento y poder, es uno de los principales desafiaos de los estudiosos de la política en la región. 


\section{Conclusiones}

Los enfoques teóricos relacionados con la transferencia de políticas públicas cada vez ganan más relevancia para el análisis de la formulación o puesta en marcha de los diferentes cursos de acción gubernamental que atienden a las situaciones socialmente problematizadas en los contextos latinoamericanos. Perspectivas teóricas en la toma de decisiones vinculadas con el racionalismo - racionalidad limitada, incrementalismo, optimo normativo, exploración mixta- y con el institucionalismo -elección racional, teoría de juegos, bote de basura, entre otros- pueden ser complementadas con los aportes de los diversos enfoques de la transferencia de políticas públicas abordados en esta revisión.

Uno de los aspectos más importantes de los enfoques trabajados es la centralidad en los actores expertos. Para complementar el entendimiento de los procesos de formulación de políticas públicas, la transferencia de políticas pública como perspectiva teórica invita a concentrar los análisis en los actores que movilizan las diferentes ideas de política a escala planetaria. Las estrategias metodológicas aportadas para el estudio de este tipo particular de actores contribuyen a su entendimiento y participación dentro de los

[ 60 ] diferentes procesos de transferencia y adaptación de políticas.

Los conceptos desarrollados dentro de esta perspectiva teórica policy transfer, policy diffusion, policy learning y policy mobilitiespermiten diferenciar tipos particulares de fenómenos relacionados con la movilización de las ideas o políticas. Esta conceptualización contribuye a la comprensión de una realidad cada vez más presente en América Latina, vinculada a la transferencia de políticas públicas.

En nuestra región, la puesta en marcha de políticas públicas cada vez más se relaciona con la transferencia de ideas provenientes de contextos foráneos. Políticas tales como la mitigación de la pobreza, la salud, los servicios públicos domiciliarios, los sistemas de transporte urbano masivo, entre otras, han sido transferidas y adaptadas a nuestros particulares contextos. Los conceptos y enfoques abordados en esta revisión contribuyen a la construcción de una perspectiva teórica que permite comprender de una mejor manera por qué decidimos transferir y adaptar decisiones de política ideadas en otros contextos. El análisis de la formulación de políticas públicas puede robustecerse incorporando el conjunto de conceptos y enfoques desarrollados. En consecuencia, las investigaciones sobre formulación 
de políticas públicas en nuestro contexto pueden beneficiarse de esta perspectiva teórica, centrando su atención en preguntas que ofrezcan una imagen más completa de la puesta en marcha de las diferentes políticas públicas, entendidas como procesos de movilización internacional de las ideas.

\section{Referencias bibliográficas}

1. Alder, Emanuel \& Hass, Peter. (1992). Conclusion: Epistemic Communities, World Order, and the Creations of a Reflective Research Program. In: Hass, Peter (ed.). Knowledge, Power and International Policy Coordination. Columbia: University of South Carolina.

2. Barbier, Marc; Cauchard, Lionel; Joly, Pierre-Benoît; Paradeise, Catherine y Vinck, Dominique. (2013). Hacia un enfoque pragmático, ecológico y político de la experticia. Revue d'anthropologie des connaissances, 7 (1). p. 1-24. DOI: 10.3917/ rac.018.i

3. Bennett, Colin \& Howlett, Michael. (1992). The Lessons of Learning: Reconciling Theories of Policy Learning and Policy Change. Policy Sciences, 25, pp. 275-294. DOI: 10.1007/BF00138786

4. Bozeman, Barry. (2000). Technology Transfer and Public Policy: A Review of Research and Theory. Research Policy, 29, pp. 627-655. DOI: 10.1016/S00487333(99)00093-1

5. Camou, Antonio. (1997). Los consejeros del príncipe. Saber técnico y política en los procesos de reforma económica en América Latina. Nueva Sociedad, 152, pp. 54-67.

6. Camou, Antonio. (2006). El saber detrás del trono: intelectuales-expertos, tanques de pensamiento y políticas económicas en la Argentina democrática (19852001). En: Garcé, Adolfo y Uña, Gerardo (comps.). Think tanks y políticas públicas en Latinoamérica: dinámicas globales y realidades regionales (pp. 139-176). Buenos Aires: Prometeo.

7. Conde, Carlos. (2005, junio 30). Policy Transfer in the EU: A Model for MENA Countries? Experts Meeting on "Approaches and Methodologies for the Assessment and Transfer of Best Practices in Governance and Public Administration». UNDESA. Túnez.

8. Corbella, Virginia y Calle, Yhelin. (2017). Políticas de transferencias monetarias condicionadas: caso Argentina y Colombia. Investigación y Desarrollo, 25 (1), pp. 135-164. DOI: 10.14482/indes.25.1.10234

9. Crivello,Silvia. (2015). UrbanPolicyMobilities:TheCaseofTurinasaSmartCity. European Planning Studies, 23, pp. 909-921. DOI: 10.1080/09654313.2014.891568 
10. Culebro, Jorge. (2008). Transferencia de políticas y construcción de lecciones para la modernización administrativa. Revista Venezolana de Gerencia, 13 (44), pp. 531-542.

11. De Jong, Martin. (1999). Institutional Transplantation: How to Adotp Good Transport Infrastructure Decision-Making Ideas from Other Countries. Delft: Eburon.

12. De Jong, Martin., Lalenis, Kostantinos. \& Mamadouh,Virginie. (2002). The Theory and Practice of Institutional Transplantation: Experiences with the Transfer of Policy Institutions. Dordrecht: Kluwer Academic. DOI: 10.1007/978-94-011-0001-4

13. Dezalay, Yves y Garth, Bryant. (2005). La internacionalización de las luchas por el poder. La competencia entre abogados y economistas por transformar los Estados latinoamericanos. México, D. F.: UNAM.

14. Dolowitz, David \& Marsh, David. (1996). Who Learns from Whom: A Review of the Policy Transfer Literature. Political Studies, 44 (2), pp. 343-357. DOI: 10.1111/j.1467-9248.1996.tb00334.x

15. Dolowitz, David \& Marsh, David. (2002). Learning from Abroad: The Role of Policy Transfer in Contemporary Policy-Making. Governance: An international Journal of Policy Administration, 13 (1), pp. 5-24. DOI: 10.1111/0952-1895.00121

16. Dolowitz, David; Hulme, Rob; Nellis, Mike \& O’Neal, Fiona. (2000). Policy Transfer and British Social Policy. Buckingham: Open University.

17. Dunlop, Claire A. (2009). Policy Transfer as Learning: Capturing Variation in what Decision-Makers Learn from Epistemic Communities. Policy Studies, 30 (3), pp.

[ 62 ] 289-311. DOI: 10.1080/01442870902863869

18. Dussauge, Mauricio. (2009). La literatura comparada sobre reformas administrativas. Desarrollos, limitaciones y posibilidades. Gestión y Política Pública, XVIII (2), pp. 439-495.

18. Dussauge, Mauricio. (2010, April 7th). From Lesson-Drawing to BoundedTransfer: Bridging Policy Transfer and Institutionalist Approaches. 14th International Research Society for Public Management Conference. The Crisis: Challenges for Public Management. Berne.

20. Evans, Mark. (2004). Policy Transfer in Global Perspective. Burlington: Ashgate.

21. Evans, Mark \& Davies, Jonathan. (1999). Understanding Policy Transfer: A Multi-Level, Multi- Disciplinary Perspective. Public Administration, 77 (2), pp. 361385. DOI: 10.1111/1467-9299.00158

22. Evetts, Julia; Mieg, Harald A. \& Felt, Ulrike. (2006). Professionalization, Scientific Expertise, and Elitism: A Sociological Perspective. In: Anders Ericsson, K.; Charness, Neil; Feltovich, Paul J. \& Hoffman, Robert R. (eds.). (2006). The Cambridge Handbook of Expertise and Expert Performance (pp. 105-126). New York: Cambridge University. DOI: 10.1017/CBO9780511816796.007 
La movilización internacional de las ideas. Enfoques, conceptos...

23. Fiszbein, Ariel et al. (2009). Transferencias monetarias condicionadas: reducción de la pobreza actual y futura. The World Bank. DOI: 10.1596/978-9-58830773-2

24. Gutiérrez, Ricardo. (2010). Between Knowledge and Politics: Experts and Water Policy Reform in Brazil. Saarbrücken: VDM.

25. Hadjiisky, Magdaléna; Pal, Leslie \& Walker, Christopher (eds.). (2017). Public Policy Transfer. Micro-Dynamics and Macro-Effects. Massachusetts: Edward Elgar. DOI: $10.4337 / 9781785368042$

26. Hall, Peter. (1993). Policy Paradigms, Social Learning and the State: The Case of Economic Policy making in Britain. Comparative Politics, 25. p, 275-297. DOI: $10.2307 / 422246$

27. Hoffman, Robert. (1998). How can Expertise Be Defined? Implications of Research from Cognitive Psychology. In: Williams, Robin; Faulkner, Wendy \& Fleck, James (eds.). Exploring Expertise (pp. 81-100). London: Palgrave Macmillan. DOI: 10.1007/978-1-349-13693-3_4

28. Huber, Bertina. (1998). Experts in Organizations: The Power of Expertise. Zurich: University of Zurich.

29. Levi-Faur, David. \& Vigoda-Gadot, Eran. (2006). New Public Policy, New Policy Transfers: Some Characteristics of a New Order in the Making. International Journal of Public Administration, 29 (4-6), pp. 247-262. DOI: 10.1080/01900690500437147

30. Marsh, David \& Rhodes, Roderick. (1992a). New Directions in the Study of Policy Networks. European Journal of Political Research, 21, pp. 181-205. DOI: 10.1111/j.1475-6765.1992.tb00294.x

31. Marsh, David \& Rhodes, Roderick. (1992b). Policy Networks in British Government.Oxford:Clarendon.DOI:10.1093/acprof:oso/9780198278528.001.0001

32. Marsh, David \& Sharman, Jason. (2009). Policy Diffusion and Policy Transfer. Policy Studies, 30 (3), pp. 269-288. DOI: 10.1080/01442870902863851

34. McCann, Eugene. \& Ward, Kevin. (2015). Thinking Through Dualisms in Urban Policy Mobilities. International Journal of Urban and Regional Research, 39 (4), pp. 828-830. DOI: 10.1111/1468-2427.12254

34. McCann, Eugene. (2017). Mobilities, Politics, and the Future: Critical Geographies of Green Urbanism. Environment and Planning A: Economy and Space, 49 (8), pp. 1816-1823. DOI: 10.1177/0308518X17708876

35. Meseguer, Covadonga \& Gilardi, Fabrizio. (2008). Reflexiones sobre el debate de la difusión de políticas. Política y Gobierno, 15 (2), pp. 315-351.

36. Morresi, Sergio y Vommaro, Gabriel. (2011). Saber lo que se hace: expertos y política en Argentina. Buenos Aires: Universidad Nacional de General Sarmiento.

37. Nickson, Andrew. (2002). Transferencia de políticas y reforma en la gestión del sector público en América Latina: el caso del New Public Management. Revista del CLAD Reforma y Democracia, 24. Recuperado de http://pdfhumanidades.com/ 
sites/default/files/apuntes/Nickson-Andrew-2002-Transferencia-de-politica-y-reformaen-el-sector-publico-de-America-Latina-El-caso-del-NPM.pdf

38. Nunn, Robin. (2008). A Network Model of Expertise. Bulletin of Science Technology \& Society, 28 (5), pp. 414-427. DOI: 10.1177/0270467608322587

39. Osorio Gonnet, Cecilia. (2015). Mecanismos de difusión de los Programas de Transferencia Condicionada en América Latina. El caso chileno. Íconos, 53, pp. 31-48. DOI: 10.17141/iconos.53.2015.1540

40. Pal, Leslie. (2006). Exporting Policy Models: The Role of International Governmental Organizations. Ottawa: Carleton University.

41. Peck, Jaime \& Theodore, Nik. (2015). Fast Policy: Experimental Statecraft at the Thresholds of Neoliberalism. Minneapolis and London: University of Minnesota. DOI: 10.5749/minnesota/9780816677306.001.0001

42. Pinilla, Juan. (2012). Think Tanks, saber experto y formación de agenda política en el Chile actual. Revista Polis, 11 (32), pp. 119-140. DOI: 10.4067/S071865682012000200008

43. Pollitt, Christopher. (2003). Public Management Reform: Reliable Knowledge and International Experience. OECD Journal on Budgeting, 3 (3), pp. 121-136. DOI: 10.1787/budget-v3-art19-en

44. Pollitt, Christopher. (2005). Desde allá hasta acá, desde ahora hasta entonces: comprando y replicando las reformas de la gestión pública. Revista del CLAD Reforma y Democracia, 31. Recuperado de http://www.redalyc.org/pdf/3575/357533663001. [ 64 ] pdf

45. Puello-Socarrás, José Francisco y Gunturiz, María Angélica. (2013). ¿Socialneoliberalismo? Organismos multilaterales, crisis global y programas de transferencia monetaria condicionada. Política y Cultura, 40, pp. 29-54.

46. Reinholde, Iveta. (2006). Policy Transfer in Public Administration: How it Works in Practice. Viešoji Politika ir Administravimas, 16, pp. 53-64.

47. Rogers, Everett. (1995). The Diffusion of Innovations. New York: Free Press.

48. Rose, Richard. (1991). What is Lesson Drawing. Journal of Public Policy, 11 (1), pp. 1-22. DOI: 10.1017/S0143814X00004918

49. Rose, Richard. (1993). Lesson-Drawing in Public Policy. New Jersey: Chatman House.

50. Rubio, Eulalia. (2002). Nuevas herramientas conceptuales para el análisis de políticas públicas: la literatura sobre difusión y transferencia de políticas. Revista GAPP, 25, pp. 23-32.

51. Sarfatti, Magali. (1988). El poder de los expertos: Ciencia y Educación de masas como fundamentos de una ideología. Revista de Educación, 285, pp. 151-189.

52. Sikkink, Katherin. (2009). The Power of Networks in International Politics. In: Kahler, Miles (ed.). Networked Politics: Agency, Power, and Governance (pp. 228247). Ithaca and London: Cornell University. DOI: 10.7591/9780801458880-013 
53. Stone, Diane. (2000). Non Governmental Policy Transfer: The Strategies of Independent Policy Institutes. International Journal of Policy and Administration, 13 (1), pp. 45-62. DOI: 10.1111/0952-1895.00123

54. Stone, Diane. (2001). Learning Lessons, Policy Transfer and the International Diffusion of Policy Ideas. University of Warwick CSGR Working Paper, 69/01. Retrieved from https://warwick.ac.uk/fac/soc/pais/research/researchcentres/csgr/ papers/workingpapers/2001/wp6901.pdf

55. Stone, Diane. (2004). Transfer Agents and Global Networks in the «Transnationalisation» of Policy. Journal of European Public Policy, 11 (3), pp. 545566. DOI: 10.1080/13501760410001694291

56. Temenos, Cristina \& Baker, Tom. (2015). Urban Policy Mobilities Research: Introduction to a Debate. International Journal of Urban and Regional Research, 39 (4), pp. 824-827. DOI: 10.1111/1468-2427.12252

57. Valencia, Enrique. (2008). Las transferencias monetarias condicionadas como política social en América Latina. Un balance: aportes, límites y debates. Annual Review of Sociology, 34, pp. 499-524. DOI: 10.1146/annurev.soc.052708.074708

58. Villatoro, Pablo. (2005). Programas de transferencias monetarias condicionadas: experiencias en América Latina. Revista de la Cepal, 86. DOI: 10.18356/383c898c-es

59. Watson, Alan. (1974). Legal Transplants: An Approach to Comparative Law. Edinburgh: Scottish Academic. 